\title{
Estimation of the Phospholipase A2 Selectivity on POPC/POPG Membranes Using the Interaction Map
}

\author{
A. S. Alekseeva ${ }^{a}$, P. E. Volynsky ${ }^{a}$, and I. A. Boldyrev ${ }^{a, *}$ \\ a Shemyakin-Ovchinnikov Institute of Bioorganic Chemistry, Russian Academy of Sciences, Moscow, 117997 Russia \\ *e-mail:ivan@lipids.ibch.ru
}

Received April 23, 2021; revised May 12, 2021; accepted May 13, 2021

\begin{abstract}
The regulation of the activity and selectivity of phospholipase A2 (PLA2), which is capable of cleaving fatty acid in the second position $(s n-2)$ of the phospholipid, is carried out through the membranebinding and catalytic sites of the enzyme. For hydrolytic activity, PLA2 must first bind to the phospholipid membrane, and the binding efficiency depends on the composition of the membrane. The membrane-binding site of PLA2 is formed by several tens of amino acids and its composition differs from enzyme to enzyme; hydrophobic and positively charged amino acids play a key role in the interaction. In this work, we investigated the interaction of PLA2 from bee venom with phospholipid bilayers of palmitoyl oleoylphosphatidylcholine (POPC) containing different amounts of palmitoyloleoylphosphatidylglycerol (POPG). On the basis of the measurements of the protein intrinsic fluorescence and the anisotropy of the fluorescence of the lipid probe we propose the construction of lipid-protein interaction maps, which reflect both the efficiency of protein binding and changes in the structure of the membrane. These changes cause alterations in the fluorescence anisotropy of the label, which in turn is a measure of the mobility of the lipid environment of the fluorescent probe. Analysis of interaction maps showed that there is a relationship between lipid mobility and enzyme binding efficiency: the optimum interaction of PLA2 with membranes from a POPC/POPG mixture lies in the region of the highest lipid mobility, and not in the region of the highest negative charge. This dependence complements the existing understanding of the process of recognition of the membrane surface by the enzyme and the selection of lipids by the enzyme already bound to the membrane. The proposed mapping method can be extended to other membrane-active proteins.
\end{abstract}

Keywords: phospholipase A2, substrate specificity, lipid-protein interaction, fluorescence spectroscopy

DOI: $10.1134 / \mathrm{S} 1990747821050032$

\section{INTRODUCTION}

Secretory phospholipase A2 (sPLA2) is an interface protein capable of hydrolyzing lipids on the surface of a biological membrane and selectively cleaving fatty acid in the second position $(s n-2)$ of a phospholipid. The protein region in contact with the membrane surface is usually called interfacial binding site, IBS. It is responsible for keeping the enzyme on the membrane surface. The catalytic site of SPLA2 is directly responsible for the hydrolysis of an individual substrate molecule. The structure of the catalytic site is highly conserved, and for various representatives of SPLA2 it has been sufficiently well studied (the key role is played by His and Asp residues in the catalytic pocket) [1]. IBS is formed by several tens of amino acids, and its structure varies from enzyme to enzyme [2]. For a representative set of human and mouse

Abbreviations: sPLA2, secretory phospholipase A2; bvPLA2, phospholipase A2 from Apis mellifera bee venom; IBS, interfacial binding site; POPC, palmitoyloleoylphosphatidylcholine; POPG, palmitoyloleoylphosphatidylglycerol; TMB-PC, 1,3,5,7tetramethyl-BODIPY-labeled phosphatidylcholine.
sPLA2 (a total of 16 different SPLA2) [3], it was shown that the hydrolysis of single-component liposomes from phosphatidylcholine, phosphatidylserine, or phosphatidylglycerol proceeds with different efficiency. In the case of liposomes made of phosphatidylcholine, the development of the reaction (the appearance of the product) occurred with a lag phase for some sPLA2, while negatively charged lipids were hydrolyzed without delay by all sPLA2. In general, the rate of liposome hydrolysis from phosphatidylglycerol was the highest. At the same time, for multicomponent liposomes from a mixture of phosphatidic acid, phosphatidylcholine, phosphatidylethanolamine, phosphatidylmethanol, phosphatidylserine and phosphatidylinositol in an equimolar ratio, lysophosphatidylcholine was found in a greater amount than other lysolipids, i.e., in a mixture of lipids, it is phosphatidylcholine that is preferentially hydrolyzed [3].

Thus, the activity and selectivity of sPLA2 is determined by the joint operation of IBS and the catalytic site. A wide range of techniques has been developed to detect the final hydrolytic activity of sPLA2 $[4,5]$, but for a more detailed study of the functioning of inter- 
face proteins a complex set of experimental approaches is employed [6]. The moment of recognition of the membrane surface by the enzyme and the process of lipid selection by the enzyme already bound to the membrane remain unclear.

To comprehend the selectivity of sPLA2, mechanisms of interaction of the enzyme with the membrane are investigated. For example, molecular dynamics was used to study the interaction of human synovial sPLA2 with membranes of various compositions [7], and it was shown that about half of the amino acids of the enzyme contact the membrane surface, providing partial immersion of the protein into the membrane. With the help of molecular dynamics in combination with mass spectrometry, it was shown that the lipid membrane works as an allosteric activator for sPLA2 [8]; binding to the membrane leads to conformational changes and activates the enzyme [9]. Using a combination of confocal microscopy and IR spectrometry, it was shown that calcium ions can be involved in the binding of sPLA2 to the membrane surface [10]; calcium ions can orient protein molecules on the membrane surface and activate the enzyme. Moreover, fluorescence microscopy and atomic force microscopy studies revealed that changes in the membrane structure caused by sPLA2 lead to an increase in the amount of adsorbed protein, that is, to the formation of new lipid binding sites for SPLA2 [11]. The reaction of the lipid bilayer to enzyme binding was also studied. For example, heterodimeric PLA2 from the venom of Nikolsky's viper induces aggregation of the lipid bilayer and the formation of multilayer lipid particles [12]. Embedding into the membrane of PLA2 from the bee venom causes the formation of depressions on the membrane surface leading to the appearance of a hydrophobic mismatch between the bilayer regions in the contact zone and beyond, which results in the displacement of phospholipid heads from the area of contact with the protein (formation of a hydrophobic spot) and thinning of the bilayer [13].

In this work, using fluorescence spectroscopy, we analyze the affinity of sPLA2 from bee venom for phospholipid bilayers consisting of neutral POPC and negatively charged POPG in various ratios. The combination of measurements of the intrinsic fluorescence of the protein and the anisotropy of the fluorescence of the lipid probe, as well as construction of interaction maps for data analysis, provides new information about the interaction of the enzyme with the membrane.

\section{MATERIALS AND METHODS}

To prepare liposomes, palmitoyloleoylphosphatidylcholine (POPC) and palmitoyloleoylphosphatidylglycerol (POPG) (Avanti Polar Lipids, USA) were used; 1,3,5,7-tetramethyl-BODIPY-labeled phosphatidylcholine (TMB-PC) was synthesized earlier [14]. Phospholipase A2 from the venom of Apis mellifera
(bvPLA2), $1.0 \mathrm{mM}$, purchased from Sigma-Aldrich, was dissolved in $0.1 \mathrm{M}$ Tris- $\mathrm{HCl}, 0.1 \mathrm{M} \mathrm{NaCl}(\mathrm{pH} 8.5)$; the enzyme concentration was evaluated by optical density at $280 \mathrm{~nm}$ using a Nano Drop spectrophotometer OneC (Thermo Fisher).

Liposomes were obtained by lipid film hydration and extrusion. Aliquots of phospholipids and a fluorescent probe were evaporated from the chloroformmethanol mixture $(2: 1)$ and dried at $7 \mathrm{~Pa}$ for at least $40 \mathrm{~min}$. The lipid film was hydrated for $2 \mathrm{~h}$ at room temperature in $0.1 \mathrm{M}$ Tris- $\mathrm{HCl}$ buffer containing $0.1 \mathrm{M} \mathrm{NaCl}, \mathrm{pH} 8.5$, and 6-10 freeze/thaw cycles (liquid nitrogen $/ 40^{\circ} \mathrm{C}$ ) were performed. Then the suspension was passed 10 times through two polycarbonate membrane filters with a pore size of $100 \mathrm{~nm}$ (Nucleopore, USA) on an Avanti mini-extruder. This method of liposome preparation allows one to obtain mainly monolamellar liposomes with a narrow size distribution [15, 16]. POPC-based liposomes containing 0, $10,15,20,25$, or $30 \mathrm{~mol} \%$ POPG with the addition of $0.025 \mathrm{~mol} \%$. TMB-PC were obtained. When the ratio of probe molecules to lipids is $1 / 4000$, no energy transfer is observed between neighboring TMB fluorophores, and the fluorescence anisotropy depends only on the fluorophore mobility (see also [17, 18]).

Protein fluorescence spectra and fluorescence anisotropy of TMB-PC were recorded on an F-4000 spectrometer (Hitachi, Japan) at $\lambda_{\mathrm{ex}}=280 \mathrm{~nm}, \lambda_{\mathrm{em}}=$ $340 \mathrm{~nm}$ and $\lambda_{\mathrm{ex}}=480 \mathrm{~nm}, \lambda_{\mathrm{em}}=510 \mathrm{~nm}$, respectively. The measurements were carried out in a $0.5 \times 0.5 \mathrm{~cm}$ quartz cuvette with constant stirring and thermostating $\left(25^{\circ} \mathrm{C}\right)$.

Fluorescence anisotropy was calculated using the formula:

$$
R=\left(I_{V V}-G I_{V H}\right) /\left(I_{V V}+2 G I_{V H}\right),
$$

where $I$ is the intensity of the fluorescence signal at different orientations of the polarizers. The orientation of the polarizers is indicated by subscripts $V$ (vertical) and $H$ (horizontal). The orientation of the polarizer first is given on the excitation side and then, on the emission side. $V H$, the polarizer on the excitation side is oriented vertically and the polarizer on the emission side is oriented horizontally; $V V$, both polarizers are oriented vertically. Factor $\mathrm{G}$ is defined as $I_{H V} / I_{H H} . H V$, the polarizer on the excitation side is oriented horizontally and the polarizer on the emission side is oriented vertically; $H H$, both polarizers are oriented horizontally.

To construct binding isotherms, fluorescence spectra of bvPLA2 $(1.2 \mu \mathrm{M})$ were recorded at varying lipid concentration in the system. The enzymatic activity of bvPLA2 was blocked by the addition of $5 \mathrm{mM}$ EDTA (for the removal of the required cofactor $\mathrm{Ca}^{2+}$ from the system). Aliquots of the liposomes suspension were added with constant stirring (final lipid concentrations $20,38,78,150,280,460$, or $800 \mu \mathrm{M}$ ) and thermostating $\left(25^{\circ} \mathrm{C}\right)$. The background signal due 
to light scattering by liposomes was subtracted from the protein emission spectra.

The maps of interactions of bvPLA2 with POPC/POPG membranes were constructed using the Scilab program. First, a three-dimensional graph was plotted, where $X$ is the protein/lipid ratio, $Y$ is the ratio of lipids in the mixture (lipid composition of the membrane), and $Z$ is $\left(F_{0}-F\right) / F_{0}$, where $F_{0}$ is the fluorescence intensity of the protein before the addition of lipids to the system and $F$, the fluorescence intensity of the protein after the addition of lipids.

Then the three-dimensional graph was transformed into a set of isolines (curves on a plane at $Z=$ const, set by the intersection of these planes with a three-dimensional experimental figure). A map of membrane changes was obtained in a similar way, but values of the membrane probe fluorescence anisotropy, $R$, were used for the $Z$ axis.

\section{RESULTS AND DISCUSSION}

\section{Binding of SPLA2 on the Membrane Surface}

The binding of bvPLA2 to liposome membranes was characterized by the intrinsic fluorescence of the enzyme with blocked enzymatic activity (due to the removal by EDTA of the necessary cofactor, $\mathrm{Ca}^{2+}$, from the system). sPLA2 from bee venom contains two tryptophan residues that change the quantum yield when the polarity of the environment changes (binding to the membrane). When a protein solution is titrated with a liposome emulsion, the intensity of the intrinsic fluorescence of the protein changes (Fig. 1a); these changes can be represented in a relative form:

$$
\left(F_{0}-F\right) / F_{0},
$$

where $F_{0}$ is the intensity of protein fluorescence before adding lipids to the system, $F$ is the intensity of protein fluorescence after the addition of lipids.

The dependence of $\left(F_{0}-F\right) / F_{0}$ changes on the lipid concentration can be used as binding isotherms to determine the affinity of sPLA2 to a certain lipid composition of the membrane (Fig. 1b). In this work, a set of bvPLA2 binding isotherms for a series of lipid mixtures from POPC/POPG was transformed into a map (Fig. 1c), where the ordinate is the protein/lipid ratio and the abscissa is the ratio of lipids in the mixture (membrane composition). The value $\left(F_{0}-F\right) / F_{0}$ is shown on the map as contour lines.

\section{Registration of Changes in the Membrane}

Monitoring the change in the state of the membrane is carried out using fluorescent lipid probes (by fluorescence anisotropy). In this case, TMB-PC (BODIPY-labeled phosphatidylcholine) is used [18], but other membrane probes can also be used. For example, for laurdan and prodan, it may be more convenient to record changes in the emission spectrum
[19]. Fluorescence anisotropy is a measure of the mobility of a fluorophore: the lower is the fluorescence anisotropy, the more mobile the probe becomes (the rotational and translational diffusion of the probe increases). In membranes, the fluorescence anisotropy of the label depends on the packing density of lipids and their mobility. The maximum value of TMB anisotropy (in propylene glycol solution at $-70^{\circ} \mathrm{C}$ ) is 0.39 , which corresponds to a completely inhibited probe [18]. Changes in the anisotropy of TMB-PC fluorescence in the lipid membrane ranges from 0.18 (gel phase) to 0.10 (liquid crystal phase) [18]. Thus, changes of anisotropy values by one hundredths reflect significant changes in the membrane lipid mobility.

On the map of membrane changes, the ordinate is the protein/lipid ratio, the abscissa is the lipid content in the mixture, and the values of fluorescence anisotropy are shown on the map as isolines (Fig. 1d). On the map of membrane changes (Fig. 1d), two minima of anisotropy were observed: at 10 and $15 \%$ POPG at protein/lipid ratios of 8 and 3 , respectively (highlighted in color). The minimum value of fluorescence anisotropy corresponds to the maximum mobility of the fluorescent probe, which is determined by the packing density of lipids around the label in the membrane.

\section{Protein Binding Versus Membrane Changes Maps}

On the protein binding map (Fig. 1c), the maximum binding was observed, which is a ridge starting at the point $(x ; y=8.5 ; 10$, the area is marked with a color). At this point, saturation of the membrane with protein is achieved. A further increase in the protein/lipid ratio does not change the value of $\left(F_{0}-F\right) / F_{0}$.

It is important that the beginning of the ridge on the map of protein binding (Fig. 1c) and the minimum on the map of membrane changes (Fig. 1d) coincide: the maximum protein binding corresponds to the maximum lipid mobility (minimum anisotropy). In this case, this condition is satisfied for liposomes containing 10\% POPG. This coincidence has a rational explanation: to accomplish it's hydrolytic activity, sPLA2 has to integrate into the membrane; for this, the protein pushes the polar lipid heads apart [13]. Accordingly, if the diffusion of lipids is limited, this is more difficult to do than in the conditions of free diffusion of lipids. In other words, the rate of lipid diffusion affects the binding constant of the protein with the membrane, and thus changes the stationary distribution of protein between solution and the membrane surface, which, in turn, determines the value of $\left(F_{0}-F\right) / F_{0}$. This example shows that not only in different phases, but also within the same phase, lipid mobility can affect the enzyme, which ultimately can determine the rate of lipid hydrolysis.

The revealed relationship between lipid mobility and enzyme binding efficiency may be a key to under- 


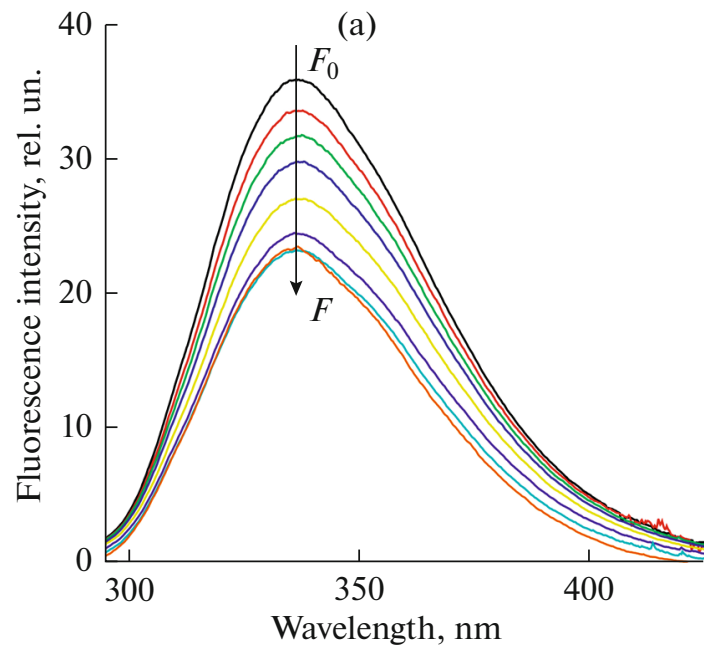

(c)

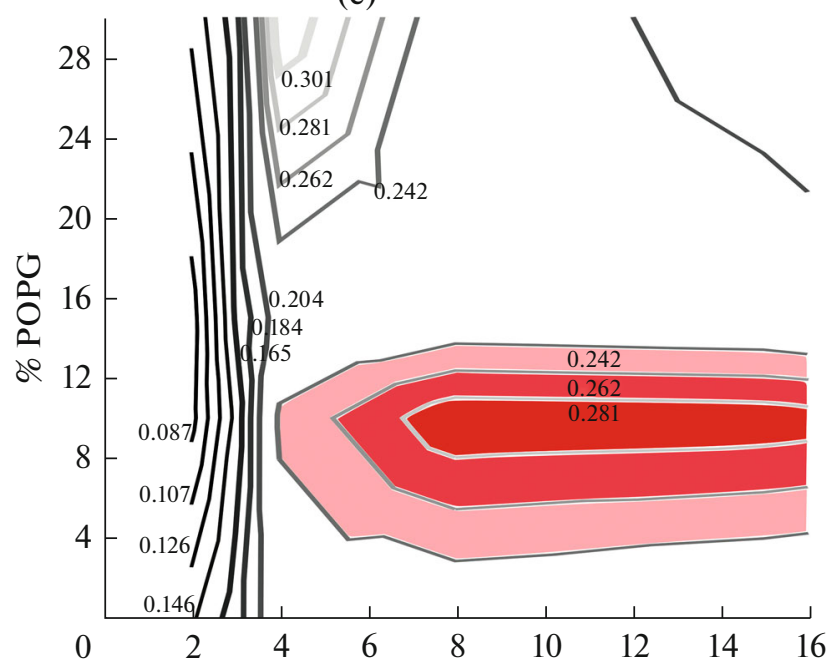

Number of bvPLA2 molecules per 1000 lipid molecules in the bilayer

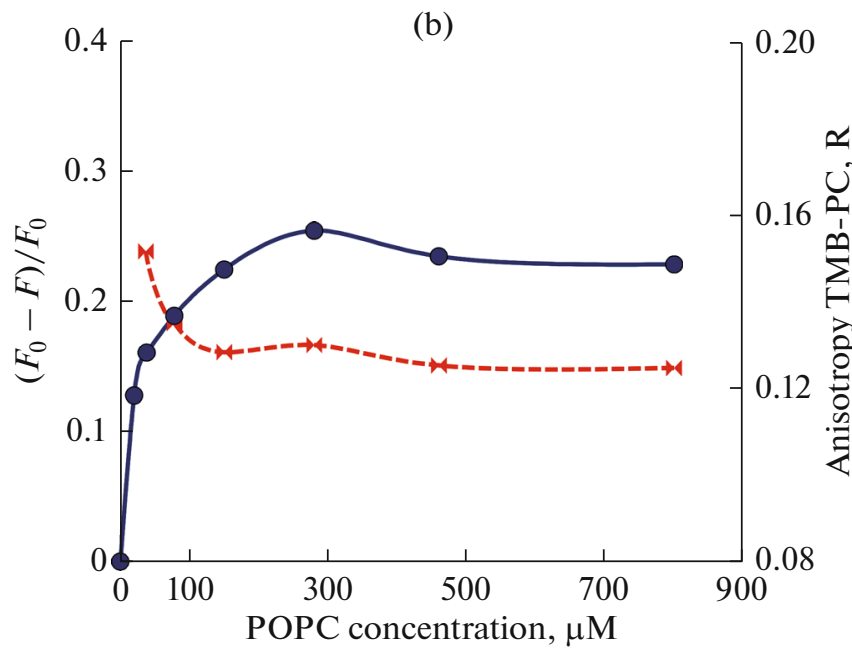

(d)

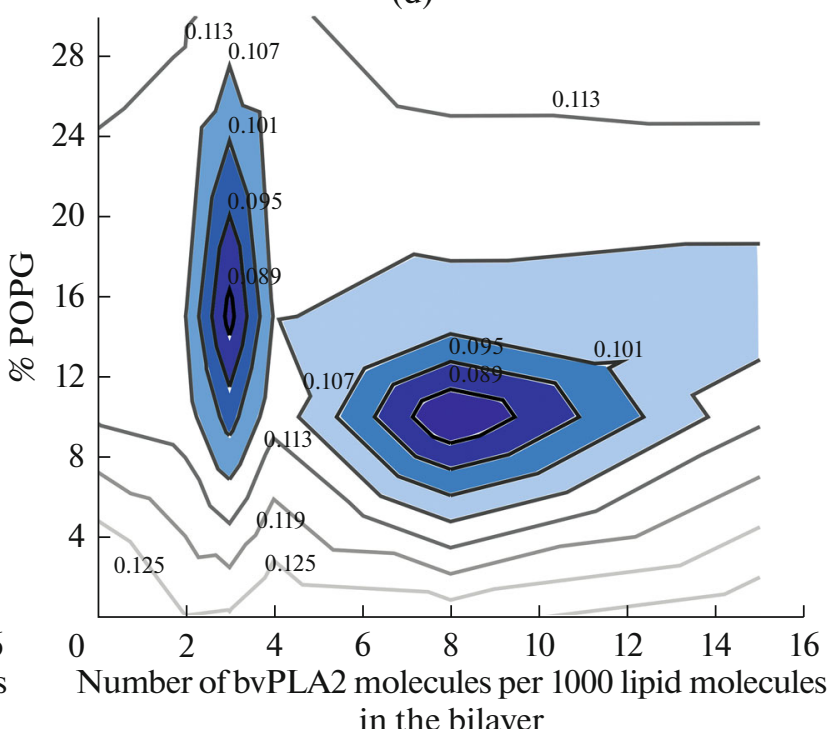

Fig. 1. Evaluation of the specificity of the interaction of sPLA2 with phospholipid membranes from POPC/POPG using the construction of interaction maps. (a) An example of a change in the intrinsic fluorescence of a protein during titration with liposome emulsion $\left(1.2 \mu \mathrm{M}\right.$ bvPLA2, $\left.\lambda_{\mathrm{ex}}=280 \mathrm{~nm}, \lambda_{\mathrm{em}}=290-450 \mathrm{~nm}\right)$; arrow shows the direction of changes with an increase in the concentration of liposomes in the system. (b) Isotherm of sPLA2 binding $\left(\lambda_{\mathrm{ex}}=280 \mathrm{~nm}, \lambda_{\mathrm{em}}=340 \mathrm{~nm}\right.$, solid line $)$ and fluorescence anisotropy of the TMB-PC membrane probe $\left(\lambda_{\mathrm{ex}}=480 \mathrm{~nm}, \lambda_{\mathrm{em}}=510 \mathrm{~nm}\right.$, dashed line). Similar curves were obtained for different liposome compositions; as an example, the binding isotherm of liposomes from POPC is shown. (c) Protein binding map.

(d) Map of membrane changes. The regions with extremum values are marked with color.

standing the substrate specificity of sPLA2 with respect to membranes of different compositions.

\section{CONCLUSIONS}

In this work, we proposed a new method for studying the binding of SPLA2 to the lipid bilayer. The method consists in constructing a map of protein binding and a map of membrane changes. The protein binding map is based on the isotherms of protein binding on lipid membranes of various compositions. The map of membrane changes is based on tracking changes in the anisotropy of the lipid probe fluorescence during protein sorption on the membrane for different lipid/protein ratios. Compar- ison of the maps revealed a relationship between lipid mobility and enzyme binding efficiency. This may be a key to understanding the substrate specificity of sPLA2 with respect to membranes of different compositions.

\section{ACKNOWLEDGMENTS}

The work was supported by the Russian Science Foundation (project no. 19-75-00101).

\section{COMPLIANCE WITH ETHICAL STANDARDS}

The authors have no obvious or potential conflicts of interest related to the publication of this article. 
This article does not contain any studies involving animals or human participants performed by any of the authors.

\section{OPEN ACCESS}

This article is licensed under a Creative Commons Attribution 4.0 International License, which permits use, sharing, adaptation, distribution and reproduction in any medium or format, as long as you give appropriate credit to the original author(s) and the source, provide a link to the Creative Commons license, and indicate if changes were made. The images or other third party material in this article are included in the article's Creative Commons license, unless indicated otherwise in a credit line to the material. If material is not included in the article's Creative Commons license and your intended use is not permitted by statutory regulation or exceeds the permitted use, you will need to obtain permission directly from the copyright holder. To view a copy of this license, visit http://creativecommons.org/licenses/by/4.0/.

\section{REFERENCES}

1. Dennis E.A., Cao J., Hsu Y.-H., Magrioti V., Kokotos G. 2011. Phospholipase A2 enzymes: Physical structure, biological function, disease implication, chemical inhibition, and therapeutic intervention. Chem. Rev. 111, 6130-6185.

https://doi.org/10.1021/cr200085w

2. Winget J.M., Pan Y.H., Bahnson B.J. 2006. The interfacial binding surface of phospholipase A2s. Biochim. Biophys. Acta. 1761, 1260-1269.

https://doi.org/10.1016/j.bbalip.2006.08.002

3. Singer A.G., Ghomashchi F., Calvez Le C., Bollinger J., Bezzine S., Rouault M., Sadilek M., Nguyen E., Lazdunski M., Lambeau G., Gelb M.H. 2002. Interfacial kinetic and binding properties of the complete set of human and mouse groups I, II, V, X, and XII secreted phospholipases A2. J. Biol. Chem. 277, 48535-48549. https://doi.org/10.1074/jbc.M205855200

4. Alekseeva A.S., Boldyrev I.A. 2020. Phospholipase A2. Activity monitoring methods. Biol. Membranes. 37, 323-336. doi 10.31857/S0233475520050035 [English version: Alekseeva A.S., Boldyrev I.A. 2020. Phospholipase A2. Methods for activity monitoring. Biochem. Moscow Suppl. Ser. A. 14 (4), 267-278.

https://doi.org/10.1134/S1990747820040030

5. Alekseeva A.S., Korotaeva A.A., Samoilova E.V., Volynsky P.E., Vodovozova E.L., Boldyrev I.A. 2014. Secretory phospholipase A2 activity in blood serum: The challenge to sense. Biochem. Biophys. Res. Commun. 454, 178-182.

https://doi.org/10.1016/j.bbrc.2014.10.069

6. Roberts M.F., Khan H.M., Goldstein R., Reuter N., Gershenson A. 2018. Search and subvert: Minimalist bacterial phosphatidylinositol-specific phospholipase C enzymes. Chem. Rev. 118, 8435-8473. https://doi.org/10.1021/acs.chemrev.8b00208

7. Qin S.S., Yu Y.X., Li Q.K., Yu Z.W. 2013. Interaction of human synovial phospholipase A2 with mixed lipid bilayers: A coarse-grain and all-atom molecular dynamics simulation study. Biochemistry. 52, 1477-1489. https://doi.org/10.1021/bi3012687
8. Mouchlis V.D., Bucher D., McCammon J.A., Dennis E.A. 2015. Membranes serve as allosteric activators of phospholipase A 2, enabling it to extract, bind, and hydrolyze phospholipid substrates. Proc. Natl. Acad. Sci. USA. 112, E516-E525.

https://doi.org/10.1073/pnas.1424651112

9. Mouchlis V.D., Chen Y., McCammon A.J., Dennis E.A. 2018. Membrane allostery and unique hydrophobic sites promote enzyme substrate specificity. J. Am. Chem. Soc. 140, 3285-3291.

https://doi.org/10.1021/jacs.7b12045

10. Kai S., Li X., Li B., Han B., Lu X. 2017. Calcium-dependent hydrolysis of supported planar lipids was triggered by honey bee venom phospholipase A2 with the right orientation at the interface. Phys. Chem. Chem. Phys. 20, 63-67.

https://doi.org/10.1039/c7cp06344j

11. Hong C.Y., Han C.T., ChaoL. 2016. Nonspecific binding domains in lipid membranes induced by phospholipase A2. Langmuir. 32, 6991-6999.

https://doi.org/10.1021/acs.langmuir.5b03915

12. Alekseeva A.S., Tretiakova D.S., Chernikov V.P., Utkin Y.N., Molotkovsky J.G., Vodovozova E.L. Boldyrev I.A. 2017. Heterodimeric V. nikolskii phospholipases A2 induce aggregation of the lipid bilayer. Toxicon. 133, 169-179.

https://doi.org/10.1016/j.toxicon.2017.05.015

13. Alekseeva A.S., Volynsky P.E., Krylov N.A., Chernikov V.P., Vodovozova E.L., Boldyrev I.A. 2021. Phospholipase A2 way to hydrolysis: Dint formation, hydrophobic mismatch, and lipid exclusion. Biochim. Biophys. Acta, Biomembr. 1863, 183481.

https://doi.org/10.1016/j.bbamem.2020.183481

14. Boldyrev I.A., Zhai X., Momsen M.M., Brockman H.L., Brown R.E., Molotkovsky J.G. 2007. New BODIPY lipid probes for fluorescence studies of membranes. J. Lipid Res. 48, 1518-1532. https://doi.org/10.1194/jlr.M600459-JLR200

15. Olson F., Hunt C.A., Szoka F.C., Vail W.J., Papahadjopoulos D. 1979. Preparation of liposomes of defined size distribution by extrusion through polycarbonate membranes. Biochim. Biophys. Acta, Biomembr. 557, 9-23. https://doi.org/10.1016/0005-2736(79)90085-3

16. Supaporn S. 2011. Effect of freeze-thawing process on the size and lamellarity of PEG-lipid liposomes. Open Colloid Sci. J. 4, 1-8. https://doi.org/10.2174/1876530001104010001

17. Sachl R., Boldyrev I.A., Johansson L.B. 2010. Localisation of BODIPY-labelled phosphatidylcholines in lipid bilayers. Phys. Chem. Chem. Phys. 12, 6027-6034. https://doi.org/10.1039/b926953c

18. Tretiakova D.S., Alekseeva A.S., Galimzyanov T.R., Boldyrev A.M, Chernyadyev A.Y., Ermakov Y.A., Batishchev O.V., Vodovozova E.L., Boldyrev I.A. 2018. Lateral stress profile and fluorescent lipid probes. FRET pair of probes that introduces minimal distortions into lipid packing, Biochim. Biophys. Acta, Biomembr. 1860, $2337-2347$. https://doi.org/10.1016/j.bbamem.2018.05.020

19. Parasassi T., Krasnowska E.K., Bagatolli L., Gratton E. 1998. Laurdan and prodan as polarity-sensitive fluorescent membrane probes. J. Fluoresc. 8, 365-373. https://doi.org/10.1023/A:1020528716621

Translated by A. Dunina-Barkovskaya 